LBNL-54195

Submitted to the

Journal of Applied Physics

\title{
Asymmetric Injection of Cathodic Arc Plasma into a Macroparticle Filter
}

\author{
André Anders and Robert A. MacGill \\ Lawrence Berkeley National Laboratory, University of California, \\ 1 Cyclotron Road, MS 53, Berkeley, California 94720-8223 \\ Original Manuscript December 11, 2003 \\ Revised Manuscript February 11, 2004
}

\author{
Corresponding Author: \\ André Anders \\ Lawrence Berkeley National Laboratory \\ 1 Cyclotron Road \\ Berkeley, CA 94720-8223, USA \\ Tel. + (510) 486-6745 \\ Fax + (510) 486-4374 \\ e-mail aanders@lbl.gov
}

This work was supported by the U.S. Department of Energy under Contract No. DEAC03-76SF00098. 


\title{
Asymmetric Injection of Cathodic Arc Plasma into a Macroparticle Filter
}

\author{
André Anders and Robert A. MacGill \\ Lawrence Berkeley National Laboratory, University of California, \\ 1 Cyclotron Road, MS 53, Berkeley, California 94720-8223
}

\begin{abstract}
The cathodic arc plasmas produced by cathode spots usually include macroparticles, which is undesirable for many applications. A common way of removing macroparticles is to use curved solenoid filters which guide the plasma from the source to the substrate. In this work, an arc source with relatively small cathode is used, limiting the possible locations of plasma production. The relative position of cathodic arc source and macroparticle filtered was systematically varied and the filtered plasma current was recorded. It was found that axis-symmetric plasma injection leads to maximum throughput only if an anode aperture was used, which limited the plasma to near-axis flow by scraping off plasma at larger angles to the axis. When the anode aperture was removed, more plasma could enter the filter. In this case, maximum filtered ion current was achieved when the plasma was injected off-axis, namely offset in the direction where the filter is curved. Such behavior was anticipated because the plasma column in the filter is known to be shifted by $\mathbf{E x B}$ and centrifugal drift as well as by non-axissymmetric components of the magnetic field in the filter entrance and exit plane. The data have implications for plasma transport variations caused by different spot locations on cathodes that are not small compared to the filter cross section.
\end{abstract}




\section{INTRODUCTION}

Cathodic arcs are known for their capability to deliver supersonic flows of fully ionized metal. They are infamous for having micron-size "macroparticles" in the plasma. Macroparticles are produced at cathode spots along with the wanted cathode plasma. Many approaches have been tried to eliminate, or at least reduce, the macroparticle content in cathodic arc plasmas. Curved magnetic filters are commonly used to separate and remove macroparticles from the cathodic arc plasma, thereby greatly improving the quality of cathodic arc thin films and coatings. Coatings without filters have macroparticles incorporated, therefore they are relatively rough and chemically not uniform, with detrimental effects on the wear and corrosion behavior.

The general idea of most macroparticle filters is to electro-magnetically guide the cathodic arc plasma to a substrate that is not in line-of-sight with the cathode, which is achieved, for example, by bending a solenoid. Due to their large inertia, the trajectories of macroparticles are practically not influenced by electric and magnetic fields, and therefore macroparticles are assumed to be lost in the filter.

Most filter designs are variations of the now-classic $90^{\circ}$ filter duct which was developed by Aksenov, Belous, Padalka, and Khoroshikh ${ }^{1,2}$ in the 1970 s. The variety of filters have been described in several reviews ${ }^{3-6}$. For applications demanding superior coatings with most complete macroparticle removal, filters with more than $90^{\circ}$ curvature and out-of-plane bent filters have been developed, either in "closed" duct architecture 7 or in open coil architecture (twisted S-filter, or "twist filter" for short ${ }^{8}$ ).

In all of these filters, plasma guiding is accomplished by a combined magnetic and electric field mechanism. The electron gyration radius is much smaller than the inner filter radius, while ion motion is only marginally affect by the magnetic field. Under these conditions, magnetic field lines are approximately equipotential lines forming an electric potential "valley" or "channel" whose minimum is near the curved filter axis. It was experimentally

shown ${ }^{9,10}$ that the potential channel has a typical depth of about $10 \mathrm{~V}$. The potential channel guides ions through the filter. The plasma is moving as a whole, remaining quasi-neutral. 
Following Khizhniyak $^{11}$, we may consider the equation of motion

$$
\frac{m_{e}}{e}\left(\frac{\partial \mathbf{v}_{e}}{\partial t}+\nabla \mathbf{v}_{e}\right)=-\left(\mathbf{E}+\mathbf{v}_{e} \times \mathbf{B}+\frac{\nabla p_{e}}{n_{e}}+\frac{\mathbf{j}}{\sigma}\right)
$$

where the bracket of the left hand side is the total derivative of the velocity, and the terms on the right hand side are acceleration by the electric field, the Lorentz force, the pressure gradient, and the electron-ion friction. The symbols are: $p_{e}=n_{e} k T_{e}$ is the electron pressure, $\mathbf{v}_{e}$ is the velocity vector of an electron fluid element. The net current term $\mathbf{j} / \sigma$ considers average electron motion relative to average ion motion and thus contains electron-ion friction; all other particle interactions are neglected. From this equation one reads that an electric field can be produced in a plasma by four factors: inertia (the term containing $\left.m_{e}\right)$, the Lorentz force $\left(\mathbf{v}_{e} \times \mathbf{B}\right.$ term), the gradient of the electron pressure, and by a net electrical current. The inertia term is very small due to the small electron mass, and also the current or ohmic term is usually small, which leads to

$$
\mathbf{E}=-\left(\mathbf{v}_{e} \times \mathbf{B}+\frac{\nabla p_{e}}{e n_{e}}\right)
$$

Outside the cathode spot region, electrons have a relatively low electron temperatures of a few $\mathrm{eV}$ and thus a small electron pressure gradient. Therefore, the electron pressure term can be neglected, and hence the remaining two terms must approximately balance:

$$
\mathbf{E} \approx-\left(\mathbf{v}_{e} \times \mathbf{B}\right)
$$

This relation shows that in order for an electric field to exist in a plasma of low electron temperature and small net current, there must exist a transverse magnetic induction, $\mathbf{B}$, and also an electron drift across $\mathbf{B}$. Because $\mathbf{E}$ is transverse $\mathbf{B}$,

$$
\mathbf{B} \cdot \mathbf{E}=-\mathbf{B} \cdot \nabla \varphi=0,
$$

where $\varphi$ is the electrostatic potential. Therefore, the electrostatic potential is a constant along a magnetic field line if the above conditions (such as low electron temperature and pressure) are fulfilled. The potential channel found in plasma filters can be associated with a set of near-axis magnetic field lines, each of which has a distinct potential, with the potential minimum approximately in the center. Such theory is known as plasma optics ${ }^{12}$. 
Electrons in crossed electric and magnetic fields are known to start an ExB drift which may lead to an off-axis shift of the plasma column ${ }^{13}$. Additionally, in a curved magnetic field, the plasma particles experience the centrifugal force, and the curvature drift appears ${ }^{13}$.

For a plasma filter of complicated geometry, such as the twist filter, characterized by a truly 3-dimensional curvature of the magnetic field lines, such drifts are difficult to compute. Worse, there is evidence ${ }^{14}$ that breaking the azimuthal symmetry in the magnetic field in the filter's entrance and exit planes due to the existence of current-carrying supply wires can cause additional offsets of the plasma flow.

The existence of plasma drifts and offsets in curved filters suggests that on-axis injection may not necessarily be the best solution in terms of maximum plasma throughput. If one could identify injection conditions that would at least partially compensate plasma offsets and drifts, perhaps better plasma transport and throughput could be achieved, and the overall performance of the coupled source-filter system may be improved. This is the idea of this work, and here we present first experimental results using asymmetric (non-axial) plasma injection into a twist filter.

\section{EXPERIMENTAL}

The overall source-filter setup is shown in Fig. 1. A water-cooled, flange-mounted cathodic arc plasma source was used similar to the one described in Ref. ${ }^{8}$. Graphite was selected as the cathode material due to its importance for the deposition of diamond-like carbon films. The important features of the plasma source included: (i) a small, circular cathode surface of $6.25 \mathrm{~mm}$ diameter on which arcs spots are allowed to burn; the cathode area was the front face of a graphite rod enclosed by an alumina ceramic tube, (ii) a manual precision cathode feed mechanism which allowed us to compensate for cathode erosion when needed (about $1 \mathrm{~mm}$ every 2000 arc pulses), and (iii) an anode consisting of copper plates that also serve as macroparticle reflectors and as plasma flow aperture.

The source was mounted horizontally injecting a jet of carbon plasma into the macroparticle filter. The horizontal position is mentioned not because horizontal or vertical positioning is important but in order to clarify the geometry of source and curved filter using words like below and above and right and left. One may use a 
Cartesian coordinate system, where the $(x, y)$ plane is the cathode plane, with $x$ being horizontal and $y$ vertical, and $Z$ is the coordinate of the normal to the cathode, i.e. in the direction of the plasma plume.

The main parts of the anode were two copper plates of $1.5 \mathrm{~mm}$ thickness, each having a central hole of $10 \mathrm{~mm}$ diameter. They were mounted to the grounded source body with 4 screws and spacers made from copper. The distance between the cathode surface and the first and second anode plate was of $\mathrm{z}=2.5 \mathrm{~mm}$ and $\mathrm{z}=10.5 \mathrm{~mm}$, respectively. The gap of $6.5 \mathrm{~mm}$ between the two anode plates represented an escape possibility for those macroparticles that were ejected from the cathode at a relative small angle with respect to the cathode surface. Since most macroparticles are ejected under shallow angles ${ }^{15}$, this anode design represents a macroparticle prefilter.

For some of the experiments described below, the second anode plate was removed. This is important because the opening of the second anode plate acted like an aperture, geometrically limiting the plasma flow. Plasma streaming through the aperture had a well-defined forward direction, however, some of the plasma produced at cathode spots was deposited on the anode, and hence less plasma was injected into the filter. When the second anode plate was removed, no aperture was obstructing plasma flow (the hole of the first anode plate was too large to be considered as an aperture).

The filter consisted of an open magnetic field coil that is bent in different planes (twist filter $\left.{ }^{8}\right)$. The filter had a total of 26 turns. It was positioned such that the first 13 turns bent the plasma flow down and the second half bent the plasma flow back horizontally to the right, as seen with the plasma flow. The filter exit plane was $75 \mathrm{~mm}$ below the injection plane. The filter coil had $32 \mathrm{~mm}$ inner diameter and $45 \mathrm{~mm}$ outer diameter. The coil material was flattened copper tubing, which provided sufficient mechanical rigidity. The filter was kept in position only by mounting the entrance turn and exit turn to a insulating mounting block. The coil had a counter-clockwise winding as seen with the plasma flow. The axial magnetic field vector was therefore essentially parallel to the plasma flow. 
In order to investigate effects of asymmetric, non-axial plasma injection, the position of the filter was varied with respect to the position of the plasma source. The plasma source with advancing cathode was flange mounted and therefore fixed in its position. We opted mounting the filter, its supporting insulator, the macroparticle "firewall" and the ion collector on one movable base plate. In this way, all of these components could be horizontally moved with respected to the source without braking vacuum. The accuracy of this motion was about $\Delta x= \pm 0.5$ $\mathrm{mm}$. The vertical position ( $y$-direction) was adjusted by venting the chamber and positioning adjustment plates under the movable base plate. Finally, the z- distance between source and filter could also be adjusted through a provision in the mounting base plate. Note that the relative position of filter and ion collector was always kept constant.

The arc plasma source was fed by a 10 -stage pulse-forming-network (PFN) of $0.5 \Omega$ impedance, as further described in Ref. ${ }^{16}$. The charging voltage was set to about $500 \mathrm{~V}$, giving a pulse amplitude of nominal $1 \mathrm{kA}$ (Fig. 2). The arc current was kept constant for all experiments. Arc plasma source and macroparticle filter were operated electrically in series. The electrical contact between source anode and filter coil was a $5 \mathrm{~cm}$ long, flexible copper braid. The flexibility was needed because the filter position was varied relative to the plasma source. The arc current was monitored using a current transformer (Pearson 0.01V/A), and the filter exit potential was measured with a Tektronix voltage probe (10 MHz bandwidth). Both signals were recorded using a digital storage oscilloscope (Tektronix TDS 744). The ion current was determined using a flat ion collector of $12 \mathrm{~cm}$ diameter positioned $10 \mathrm{~cm}$ from the macroparticle "firewall." The negative bias of the ion collector was $-90 \mathrm{~V}$ with respected to ground. With this bias, the collector worked like a plane Langmuir probe in the ion saturation branch; secondary electron emission is still negligible. The collector bias was kept constant during each pulse by using a $1500 \mu \mathrm{F}$ capacitor. The ion current signal was obtained via a current transformer (Pearson $0.1 \mathrm{~V} / \mathrm{A}$ ), which was also recorded at the digital storage oscilloscope.

Although the arc current has some flat portion, the ion current showed noticeable deviation from a rectangular pulse shape. Therefore, rather than using nominal current amplitudes for evaluation, which are ill defined with such pulse shape, we used the charge per pulse, i.e. $\int I d t$, where the integration is over the pulse duration. The performance of the source-filter system can judged by system coefficient defined as 


$$
\kappa_{\text {pulse }}=\int_{0}^{t_{\text {pulse }}} I_{i}(t) d t / \int_{0}^{t_{\text {pulse }}} I_{\text {arc }}(t) d t .
$$

Due to the explosive nature of plasma production and associated fluctuating ion current signals, each recorded data point was averaged over 16 individual arc pulses.

The cathodic arc vacuum chamber was cryogenically pumped with a base pressure of about $5 \times 10^{-5} \mathrm{~Pa}$; the pressure during operation was in the mid $10^{-4} \mathrm{~Pa}$ range.

\section{RESULTS AND DISCUSSION}

In a first series of experiments, both anode plates were used (in Figure 3 labeled "with anode aperture"), while in another series the second anode plate was removed (labeled "without anode aperture"). The relative source-filter position was systematically scanned ( $x$ in the range $+10 \mathrm{~mm}$ to $-10 \mathrm{~mm}, y=-6 \mathrm{~mm}, 0,6 \mathrm{~mm}$, and $z=10 \mathrm{~mm}, 22$ $\mathrm{mm}$ ). The results are compiled in Figure 3. Data are presented as integrated filtered ion current, i.e. as the ion charge per pulse collected by the biased ion collector. The system coefficient can be determined using these data and the constant arc charge per pulsed, which was $\int I_{\text {arc }} d t=660 \mathrm{mAs}$.

In the case when the anode aperture was utilized, the plasma plume was well defined but less plasma entered the filter, which becomes immediately clear when comparing the absolute values of the ion charge collected. Note that in Figure 3, the same scale was used for all situations investigated, so comparison is readily possible. In contrast, with the anode aperture, much more plasma can enter the filter, and also more plasma has been detected leaving the filter.

From the data shown in Figure 3 one can draw a number of conclusions. When the plasma is injected with a wellcollimated beam, achieved by "scraping off" plasma streaming under a large angle to the filter entrance axis, the best transport is observed for near axis injection $(x=0, y=0)$. However, when we allowed not only near-axis plasma but plasma flow with greater angle to the entrance exit, more plasma in total is injected, which "skews" the overall transport in such a way that off-axis, asymmetric injection resulted in the greatest filtered ion charge per pulse. Therefore, there is no simple conclusion whether or not off-axis, asymmetric injection could be used to 
at least partially offset plasma drifts occurring in the curved filter. The result depends on the anode configuration. If one wanted to maximize the filtered ion current or charge per pulse, one would use an anode that obstructs as little as possible the flow of plasma towards the filter entrance. The experimental data show that in this situation, off-axis injection can result in greater filtered ion current. For the specific case investigated here, the maximum filtered ion current was obtained when the off-axis injection was to the right as seen with the plasma flow, i.e. to the direction where the filter coil is going to curve to) and the bottom, i.e. also the direction where the filter is going to curve to.

In terms of absolute plasma throughput one considers best the system coefficient which describes the combined source and filter system. From Figures 2 and 3 one recognizes that the system coefficient is of order $1 \%$ in the current experiments, which is not unusual for a curved filters, and especially double-bent filters. One should recall that as a rule of thumb, the maximum (unfiltered) ion current is about $10 \%$ of the arc current. While the system coefficient is not very high, one should recognize that the plasma throughput has only be studied and optimized with respect to the plasma injection location, while other means of optimizations, such as using optimum filter bias (near 20 Volts) with a "self-bias-resistor", Ref. ${ }^{10}$, have not been utilized. Figure 2 shows also the electric potential of the filter exit, which indicates that (i) there is a large variation of the filter potential due to the time-dependent current flowing in the filter coil and due to the inductive component of the voltage drop, and (ii) the plasma throughput falls dramatically near at the end of the arc pulse due to the lack of sufficient positive filter potential. The latter is due to the negative value of $d I / d t$. The filter potential is therefore reduced, and the filter's potential channel is too shallow to provide satisfactory ion transport.

The present experiment on plasma injection has implications on plasma transport in filters where the cathode size is not much smaller than the filter cross section. In these cases, the cathode spot can "travel" on a relatively large cathode surface and cause great variation in terms of off-axis plasma injection into the filter. That is, even when the amount of plasma produced at a cathode spot was constant in time, the variation in injection location would cause a different amount of plasma being transported in the filter, with the rest being lost in the filter. Ideally, one would use a small-area cathode, with "small" understood in relation to the filter's cross section, and one would position the cathode somewhat off-axis as described in the present experiments. Such approach could result in 
noticeable improvements of the system coefficient, i.e. the overall filtered plasma for a given amount of plasma produced by the cathodic arc discharge.

\section{SUMMARY AND CONCLUSIONS}

It was experimentally shown that the way of carbon plasma injection into a twist filter (i.e. an open-coil, S-shaped filter that is bent out of the S-plane) can affect quite noticeably the filtered plasma output, depending on the shape of the anode and on the relative source-filter position. If the anode is built such as to only allow a fraction of the plasma to move as a narrow plume towards the filter entrance, best plasma transport through the filter is observed when the plasma injection is close to the entrance axis and the cathode surface is not too far from the filters magnetic field. When the plasma is less plumed, though, e.g. when using an anode that does not scrape off much of the plasma flow, maximum throughput through the filter is obtained when the plume is not injected on axis but slightly offset towards the curvature of the twist filter. This effect may be related to plasma drifts known to occur in crossed electric and magnetic fields, and especially when curved fields are involved, as it is in our case. Additionally, one should realize that an azimuthal field asymmetry, which is due to the presence of currentcarrying wires at filter entrance and exit, may contribute to the effect.

\section{ACKNOWLEDGEMENT}

This work was supported by the U.S. Department of Energy under Contract No. DE-AC03-76SF00098. 


\section{REFERENCES}

1 I. I. Aksenov, V. A. Belous, V. G. Padalka, and V. M. Khoroshikh, Sov. J. Plasma Phys. 4, 425-428 (1978).

2 I. I. Aksenov, V. A. Belous, V. G. Padalka, and V. M. Khoroshikh, Instrum. Exp. Tech. 21, 1416-1418 (1978).

3 R. L. Boxman and S. Goldsmith, Surf. Coat. Technol. 52, 39-50 (1992).

4 R. L. Boxman, V. Zhitomirsky, B. Alterkop, E. Gidalevitch, I. Beilis, M. Keidar, and S. Goldsmith, Surf. Coat. Technol. 86-87, 243-253 (1996).

5 I. G. Brown, Annual Rev. Mat. Sci. 28, 243-269 (1998).

$6 \quad$ A. Anders, Surf. Coat. Technol. 120-121, 319-330 (1999).

$7 \quad$ X. Shi, B. K. Tay, H. S. Tan, E. Liu, J. Shi, L. K. Cheah, and X.Jin, Thin Solid Films 345, 1-6 (1999).

8 A. Anders and R. A. MacGill, Surf. Coat. Technol. 133-134, 96-100 (2000).

9 M. M. M. Bilek, Y. Yin, and D. R. McKenzie, IEEE Trans. Plasma Sci. 24, 1165-1173 (1996).

E. Byon and A. Anders, J. Appl. Phys. 93, 8890-8897 (2003).

11 N. A. Khizhniyak, Sov. Phys. Tech. Phys. 10, 655-661 (1965).

12 A. I. Morozov and S. V. Lebedev, in Reviews of Plasma Physics, Vol. 8, edited by M. A. Leontovich (Consultants Bureau, New York, 1980), p. 301-460.

13 F. F. Chen, Plasma Physics and Controlled Fusion (Plenum Press, New York, 1984).

14 M. M. M. Bilek and A. Anders, Plasma Sources Sci. Technol. 8, 488-493 (1999).

15 J. E. Daalder, J. Phys. D: Appl. Phys. 9, 2379-2395 (1976).

16 A. Anders, R. A. MacGill, and T. A. McVeigh, Rev. Sci. Instrum. 70, 4532-4534 (1999). 


\section{FIGURE CAPTIONS}

FIG. 1

Schematic of the overall source-filter setup. The cathodic arc plasma source is flange-mounted and fixed. The twist filter, macroparticle "firewall" and ion collector could be moved together with respect to the arc source because they were mounted on a movable base plate.

FIG. 2

Example of an arc current pulse, filtered carbon ion current, and electrical potential measured at the filter exit. The charging voltage was set to about $500 \mathrm{~V}$, giving an arc current amplitude of nominal $1 \mathrm{kA}$.

\section{FIG. 3}

Ion charge per pulse collected by the negatively biased ion collector. The data were obtained for systematically varied positions of the plasma source with respect to the filter entrance (horizontal $x$ in the range $+10 \mathrm{~mm}$ to -10 $\mathrm{mm}$, vertical $y=-6 \mathrm{~mm}, 0,6 \mathrm{~mm}$, and longitudinal $z=10 \mathrm{~mm}, 22 \mathrm{~mm}$ ). Each data point is the average of 16 arc pulses. 


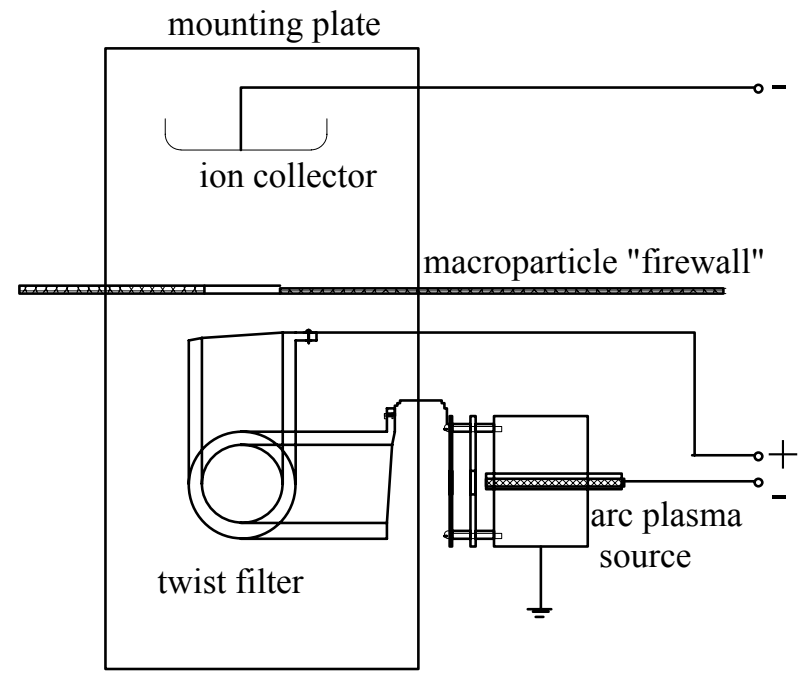

FIG. 1 


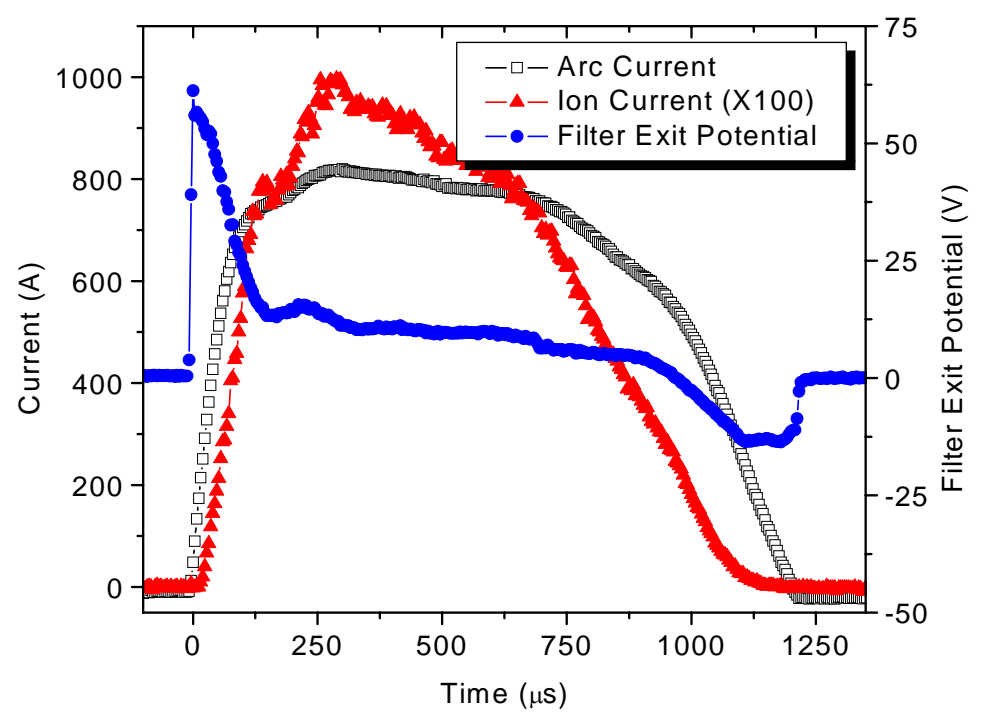

FIG. 2 

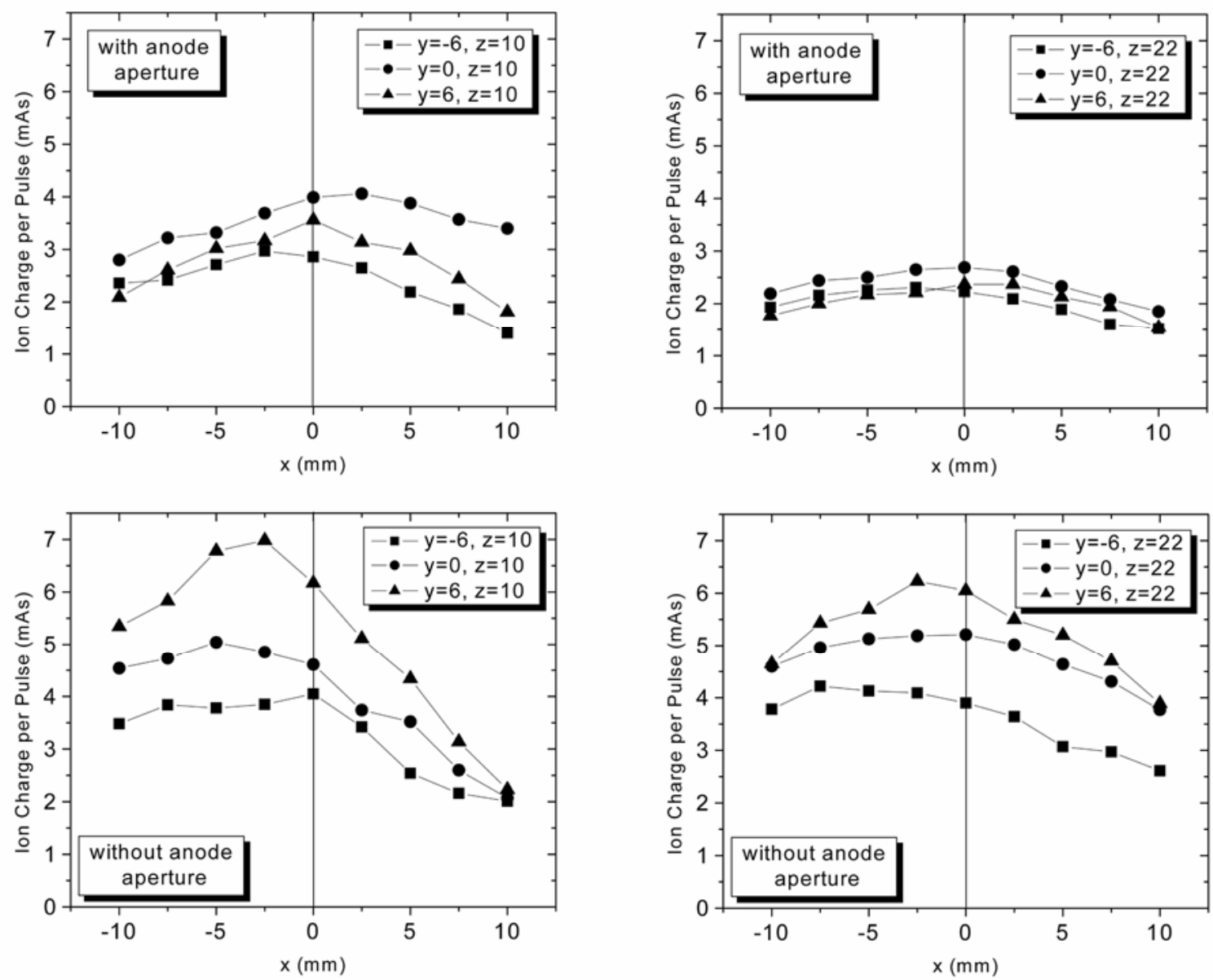

FIG. 3 